ESAIM: PROCEEDINGS AND SURVEYS, December 2017, Vol. 58, p. 27-39

Stéphane DELLACHERIE, Gloria FACCANONI, Bérénice GREC, Frédéric LAGOUTIERE, Yohan PENEL

\title{
A LOW-MACH ROE-TYPE SOLVER FOR THE EULER EQUATIONS ALLOWING FOR GRAVITY SOURCE TERMS*
}

\author{
Wasilij Barsukow ${ }^{1}$, Philipp V.F. Edelmann ${ }^{2}$, Christian Klingenberg ${ }^{1}$ and \\ FRIEDRICH K. RÖPKE ${ }^{2,3}$
}

\begin{abstract}
In order to perform simulations of low Mach number flow in presence of gravity the technique from [23] is found insufficient as it is unable to cope with the presence of a hydrostatic equilibrium. Instead, a new modification of the diffusion matrix in the context of Roe-type schemes is suggested. We show that without gravity it is able to resolve the incompressible limit, and does not violate the conditions of hydrostatic equilibrium when gravity is present. These properties are verified by performing a formal asymptotic analysis of the scheme. Furthermore, we study its von Neumann stability when subject to explicit time integration and demonstrate its abilities on numerical examples.
\end{abstract}

\section{INTRODUCTION}

The Euler equations

$$
\begin{aligned}
\partial_{t} \varrho+\nabla \cdot(\varrho \mathbf{v}) & =0 \\
\partial_{t}(\varrho \mathbf{v})+\nabla \cdot(\varrho \mathbf{v} \otimes \mathbf{v}+p \cdot \mathbb{1}) & =0 \\
\partial_{t} E+\nabla \cdot[\mathbf{v}(E+p)] & =0
\end{aligned}
$$

are a model of the motion of an inviscid fluid. Therefore a solution of these equations is of relevance whenever viscosity and further effects are negligible. Reliable numerical methods are important in view of the complexity of the equations. This paper focuses on finite volume schemes. They discretize space as a mesh and interprete the discrete values as volume averages over each cell. Given fluxes through the boundaries this yields an explicit update procedure which inherently reflects the nature of (1)-(3) as a system of conservation laws. Such timeexplicit integration restricts the choice of the time step to small values, such that waves from one interface have not yet reached the other interface in one time step (CFL condition [7]). If these waves travel very fast compared to the speed of the flow, one might consider the method to be unefficient. Time-implicit methods do not have a condition on the time step other than desired accuracy, but require the solution of large (nonlinear) systems of equations at every time step. Whether an implicit time integration will yield results of comparable accuracy in less time depends on the particular problem and the implementation. For fluid flow it seems that typical implicit methods are more efficient if the signal speed is about 100 times larger than the flow velocity.

\footnotetext{
* WB acknowledges support by the German National Academic Foundation. The work of FKR and PVFE is supported by the Klaus Tschira Foundation. CK acknowledges support by the DFG SPP "Software for exascale computing".

${ }^{1}$ Department of Mathematics, Würzburg University, Germany

2 Heidelberg Institute for Theoretical Studies, Germany

${ }^{3}$ Heidelberg University, Germany
}

(C) EDP Sciences, SMAI 2017 
The choice of the interface flux should assure upwinding for supersonic flows. Additionally, it should introduce enough viscosity for the scheme to be able to dissipate entropy as physically required in shock waves, but at the same time not introduce too much of it to avoid excessive smearing of shocks and other features of the flow. Though a scheme is in the end an update procedure that produces discrete values out of a set of discrete values, in the intermediate step one can introduce a continuous function whose cell averages agree with the given ones (reconstruction). Computing the flux through the boundary out of the exact time evolution of this data can be a successful definition of the boundary flux ([10]), but usually turns out to be difficult to compute for nonlinear systems and/or reconstructions of higher order. Also the reconstruction and averaging procedures introduce so much numerical error that an exact solution of an intermediate step does not necessarily make the overall scheme superior to others. Therefore simpler definitions of boundary fluxes are studied $([5,13,21,25]$ for example), in order to construct methods that are robust, but computationally less expensive.

Optimally, numerical methods should be reproducing the main features of an exact solution. In principle, if at all, this can only be guaranteed in the limit of fine resolutions, which however is impractical, as true simulations are always run at some finite scale of cell size. At the same time, in experiments one observes that on the same grid certain methods are able to reproduce the exact solution of some test problem to much higher accuracy than others. Sometimes this can be related to the (formal) convergence rate of a method. Typically, performing the reconstruction on a function space of higher dimensionality, one finds the scheme to be formally a higher order approximation (in space) to the original equations. Performing the integration in time with a method of higher order analogously improves the formal order of the method in time.

However, for certain setups higher order alone need not be sufficient (nor actually necessary) to improve the quality of the numerical solution at finite resolution. Special care can be taken with regard to certain, sufficiently "important" special cases. Their properties can be then taken into account in the numerical method while still having a generally aplicable scheme. In this paper, one such special setup is considered to be the low Mach number limit of ideal hydrodynamics. It shows the transition from compressible to incompressible hydrodynamics for flows, where the sound speed is much higher than the speed of the fluid flow (see Section 1). Such flow regions are ubiquitous in practical everyday life applications, but can occur also in wakes of supersonically moving objects, or in astrophysical applications, such as interiors of stars. Whereas a uniformly nearly-incompressible flow suggests the use of a method that directly solves the equations of incompressible hyrodynamics, the mentioned examples have regions of slow flow coexisting with regions where the compressible nature of the fluid cannot be ignored.

When gravity is present, the Euler equations (1)-(3) have to be augmented by source terms:

$$
\begin{aligned}
\partial_{t} \varrho+\nabla \cdot(\varrho \mathbf{v}) & =0 \\
\partial_{t}(\varrho \mathbf{v})+\nabla \cdot(\varrho \mathbf{v} \otimes \mathbf{v}+p \cdot \mathbb{1}) & =\varrho \mathbf{g} \\
\partial_{t} E+\nabla \cdot[\mathbf{v}(E+p)] & =\varrho \mathbf{v} \cdot \mathbf{g}
\end{aligned}
$$

with $\mathbf{g}$ a given, possibly spatially varying gravitational acceleration. In the limit of small Mach numbers, a formal asymptotic analysis is performed in Section 2. Having gravity balancing the pressure gradient of the gas leads to hydrostatic equilibria, which describe stratifications of atmospheres or regions in the interiors of stars. The main difference to the limit in the homogeneous case is the persistent compressible nature of the gas. Roughly speaking, even for low Mach numbers, gas tends to form the stable configuration of being compressed and denser in the direction of gravity.

There exist a certain number of numerical methods that are able to resolve the limit of low Mach numbers in the homogeneous case (1)-(3). The analytical results indicate a decoupling of sound waves and fluid flow in the limit. There exists a number of approaches to treat the limit numerically, e.g. [6,8,16]. It has been found by [23] that well-chosen modifications of the diffusion matrix in Roe-type schemes might also lead to a very much improved behaviour of numerical solutions of slow flows. This has been confirmed in [12]. They are independent of the way the time integration is performed, and can be used with both implicit and explicit methods. However the particular modification that has been used so far is incompatible with the presence of 
a hydrostatic equilibrium when applied to (4)-(6). The scheme proposed by [19] and analyzed in this paper therefore is a modification which still is able to resolve the incompressible limit in the homogeneous case (1)-(3), but which is consistent with the presence of gravity. In Section 3.2 we describe the strategy and present and analyze our scheme in Section 3.3. Integration in time and the associated question of stability is addressed in Section 4. Section 5 exemplifies applications of the method.

\section{THE LOW MACH NUMBER LIMIT}

\subsection{Rescaled equations}

To study the limit of low Mach numbers for the homogeneous Euler equations (1)-(3), one introduces a family of solutions, parametrized by a real number $M>0$, such that the local Mach number

$$
M_{\mathrm{loc}}(\mathbf{x}, t):=\frac{|\mathbf{v}(\mathbf{x}, t)|}{\sqrt{\frac{\gamma p(\mathbf{x}, t)}{\varrho(\mathbf{x}, t)}}}
$$

is asymptotically proportional to this parameter: $M_{\text {loc }} \in \mathcal{O}(M)$ when $M \rightarrow 0$. The notation $f \in \mathcal{O}(M)$ means that if $f$ is expanded as a power series in $M$, then the highest order term is proportional to $M: f=$ $M f^{(1)}+M^{2} f^{(2)}+\ldots$ Analogously $f \in \mathcal{O}(1)$ means that $f$ tends to a constant when $M \rightarrow 0$, i.e. $f=$ $f^{(0)}+M f^{(1)}+M^{2} f^{(2)}+\ldots$

Every member of the family fulfills the same equation of state

$$
E=\frac{p}{\gamma-1}+\frac{1}{2} \varrho|\mathbf{v}|^{2}
$$

The most general asymptotic scalings are

$$
\begin{aligned}
\mathbf{x} & =M^{\mathfrak{a} \tilde{\mathbf{x}}}, & t & =M^{\mathfrak{b}} \tilde{t}, \\
\varrho(\mathbf{x}, t) & =M^{\mathfrak{c}+2-2 \mathfrak{d}} \tilde{\varrho}(\tilde{\mathbf{x}}, \tilde{t}), & \mathbf{v}(\mathbf{x}, t) & =M^{\mathfrak{d}} \tilde{\mathbf{v}}(\tilde{\mathbf{x}}, \tilde{t}), \\
E(\mathbf{x}, t) & =M^{\mathfrak{c}} \tilde{E}(\tilde{\mathbf{x}}, \tilde{t}), & p(\mathbf{x}, t) & =M^{\mathfrak{c}} \tilde{p}(\tilde{\mathbf{x}}, \tilde{t}),
\end{aligned}
$$

with so far no further conditions on $\mathfrak{a}, \mathfrak{b}, \mathfrak{c}, \mathfrak{d}$, as can be found from a direct computation. Note that quantities with a tilde are taken to be $\mathcal{O}(1)$ when expanded as power series in $M$.

Furthermore every member of the family shall be a solution of the Euler equations. Inserting the above scalings yields a system of equations that is fulfilled by quantities with a tilde. These equations will be called rescaled, and are not the Euler equations again, because the Mach number changes. They are found to be

$$
\tilde{E}=\frac{\tilde{p}}{\gamma-1}+\frac{1}{2} M^{2} \tilde{\varrho}|\tilde{\mathbf{v}}|^{2}
$$

and

$$
\begin{aligned}
& M^{\mathfrak{a}-\mathfrak{d}-\mathfrak{b}} \partial_{t} \tilde{\varrho}+\nabla \cdot(\tilde{\varrho} \tilde{\mathbf{v}})=0, \\
& M^{\mathfrak{a}-\mathfrak{d}-\mathfrak{b}} \partial_{t}(\tilde{\varrho} \tilde{\mathbf{v}})+\nabla \cdot\left(\tilde{\varrho} \tilde{\mathbf{v}} \otimes \tilde{\mathbf{v}}+\frac{\tilde{p}}{M^{2}} \cdot \mathbb{1}\right)=0, \\
& M^{\mathfrak{a}-\mathfrak{d}-\mathfrak{b}} \partial_{t} \tilde{E}+\nabla \cdot(\tilde{\mathbf{v}}(\tilde{E}+\tilde{p}))=0 .
\end{aligned}
$$

The factor in front of the time derivatives is related to the dimensionless Strouhal number

$$
S t r_{\mathrm{loc}}=\frac{x}{|\mathbf{v}| t}=\frac{M^{\mathfrak{a}} \tilde{x}}{M^{\mathfrak{d}} \tilde{\mathbf{v}} \cdot M^{\mathfrak{b}} \tilde{t}} .
$$


This factor is not identical to the Strouhal number, but is just its asymptotic $M$-scaling. As an additional condition on the family of solutions one imposes $\operatorname{Str} \in \mathcal{O}(1)$, i.e. $\mathfrak{a}-\mathfrak{d}-\mathfrak{b}=0$. This corresponds to an adaptation of the time scales to the speed of the fluid rather than to domain crossing times of sound waves. Note that in this case the precise value of $\mathfrak{a}, \mathfrak{b}, \mathfrak{c}, \mathfrak{d}$ does not matter any more for the form of the rescaled equations. We drop the tilde in what follows to simplify notation.

\subsection{The incompressible limit}

The solutions to

$$
\begin{aligned}
\partial_{t} \varrho+\nabla \cdot(\varrho \mathbf{v}) & =0 \\
\partial_{t}(\varrho \mathbf{v})+\nabla \cdot\left(\varrho \mathbf{v} \otimes \mathbf{v}+\frac{p}{M^{2}} \cdot \mathbb{1}\right) & =0 \\
\partial_{t} E+\nabla \cdot[\mathbf{v}(E+p)] & =0
\end{aligned}
$$

tend to solutions of the incompressible Euler equations in the limit of low Mach numbers $([1,9,14,15,17,18,22,24]$ and others). This can formally be seen by expanding all quantities as series in $M$, e.g. for the pressure

$$
p(x, t)=p^{(0)}(x, t)+M p^{(1)}(x, t)+M^{2} p^{(2)}(x, t)+\mathcal{O}\left(M^{3}\right) .
$$

Inserting these into the above equations, collecting order by order and assuming impermeable boundaries gives

$$
\begin{aligned}
p^{(0)} & =\mathrm{const}, \\
p^{(1)} & =\mathrm{const}, \\
(\nabla \cdot \mathbf{v})^{(0)} & =0
\end{aligned}
$$

and

$$
\begin{aligned}
\partial_{t} \varrho^{(0)}+\mathbf{v}^{(0)} \cdot \nabla \varrho^{(0)} & =0, \\
\partial_{t} \mathbf{v}^{(0)}+\left(\mathbf{v}^{(0)} \cdot \nabla\right) \mathbf{v}^{(0)}+\nabla p^{(2)} / \varrho^{(0)} & =0 .
\end{aligned}
$$

These equations describe incompressible flows. Conditions (21), (22) and (23) are constraints for the solution at any time. Initial data that fulfill them are called well-prepared. Not well-prepared initial data may lead to an incompressible flow as well, but then an initial disturbance is produced.

To summarize, if the initial data for the compressible, homogeneous Euler equations are chosen to have spatial pressure fluctuations scale with $\mathcal{O}\left(M^{2}\right)$ and the divergence of the velocity field scale with $\mathcal{O}(M)$, then the solution converges to the solution of the incompressible Euler equations in the limit $M \rightarrow 0$, with only these pressure fluctuations playing the role of the dynamic pressure.

\subsection{Kinetic energy}

The equation for the kinetic energy density $E_{\text {kin }}=\frac{1}{2} \varrho|\mathbf{v}|^{2}$ is found to be

$$
\partial_{t} E_{\text {kin }}+\nabla \cdot\left[\mathbf{v}\left(E_{\text {kin }}+\frac{p}{M^{2}}\right)\right]=\frac{p}{M^{2}} \nabla \cdot \mathbf{v}
$$

The source term vanishes for incompressible flows and in this case the kinetic energy becomes a conserved quantity. For compressible flows, this is true in the limit $M \rightarrow 0$ as well, despite of $\frac{\nabla \cdot v}{M^{2}} \notin \mathcal{O}(M)$. Expanding the quantities and using (21) and (22) makes the terms proportional to $\frac{1}{M}$ or $\frac{1}{M^{2}}$ cancel and gives

$$
\partial_{t} E_{\text {kin }}+\nabla \cdot\left[\mathbf{v}\left(E_{\text {kin }}+p^{(2)}\right)\right]=p^{(2)} \nabla \cdot \mathbf{v}+\mathcal{O}(M) .
$$


Now the source term indeed is $\mathcal{O}(M)$ and vanishes in the limit $M \rightarrow 0$. The total kinetic energy is conserved for solutions to the compressible, homogeneous Euler equations in the low Mach number limit.

\section{Hydrostatic EQUilibria}

In presence of an exterior gravitational field, equations (4)-(6) describe the motion and the steady states of the fluid. A possible application is the interior of a star, or an atmosphere, whose state at rest is then given by just

$$
\nabla p=\varrho \mathbf{g}
$$

This equation leads to a solution once a relationship between $p$ and $\varrho$ is known. In view of $p=$ const $\varrho \varrho T$ typically this hydrostatic equilibrium can be determined, once the temperature of the gas is given everywhere. Note that this equilibrium can be unstable. Having buoyantly lighter fluid placed under heavier one, small perturbations will increase and ultimately lead to convective motion and mixing of the two fluids. The reversed situation is a stable one, and therefore small perturbations will not have such a dramatic effect.

In applications, one is interested in resolving this motion of a stratified gas numerically. Given a stratification, the local Mach number $|\mathbf{v}| / \sqrt{\gamma T}$ of this motion becomes small for small velocities.

Rescaling gravity as $\mathbf{g}=M^{\mathfrak{f}} \tilde{\mathbf{g}}$, and choosing the Strouhal number not to vary with $M$ again, one arrives at the rescaled system

$$
\begin{aligned}
\partial_{t} \varrho+\nabla \cdot(\varrho \mathbf{v}) & =0 \\
\partial_{t}(\varrho \mathbf{v})+\nabla \cdot\left(\varrho \mathbf{v} \otimes \mathbf{v}+\frac{p}{M^{2}} \cdot \mathbb{1}\right) & =\frac{1}{F r^{2}} \varrho \mathbf{g} \\
\partial_{t} E+\nabla \cdot[\mathbf{v}(E+p)] & =\frac{M^{2}}{F r^{2}} \varrho \mathbf{v} \cdot \mathbf{g}
\end{aligned}
$$

Again, the tilde has been dropped. Here $F r=M^{\mathfrak{d}-\frac{a+f}{2}}$ is the power of $M$ which appears in the definition of the local Froude number

$$
F r_{\text {loc }}=\frac{|\mathbf{v}|}{\sqrt{|\mathbf{x} \cdot \mathbf{g}|}}=M^{\mathfrak{d}-\frac{a+f}{2}} \frac{|\tilde{\mathbf{v}}|}{\sqrt{|\tilde{\mathbf{x}} \cdot \tilde{\mathbf{g}}|}} .
$$

Note that whereas the Mach number (squared) is the ratio between kinetic and internal energies, the Froude number (squared) quantifies the relative magnitudes of kinetic and gravitational potential energies. In the example of a constant gravity field, this gives the appearance of the independent spatial variable in the definition of $F r_{\text {loc }}$ the interpretation of the height measured in the direction opposite to $\mathrm{g}$.

Having motions with a small velocity superposing an $M$-independent background suggests to actually choose the scalings to be $\mathfrak{a}=\mathfrak{f}=0$ and $\mathfrak{d}=1$ :

$$
\begin{array}{rlrl}
\mathbf{x} & =\tilde{\mathbf{x}}, & t & =M^{\mathfrak{b}} \tilde{t}, \\
\varrho(\mathbf{x}, t) & =M^{\mathfrak{c}} \tilde{\varrho}(\tilde{\mathbf{x}}, \tilde{t}), & \mathbf{v}(\mathbf{x}, t) & =M^{\tilde{\mathbf{v}}}(\tilde{\mathbf{x}}, \tilde{t}), \\
E(\mathbf{x}, t) & =M^{\mathfrak{c}} \tilde{E}(\tilde{\mathbf{x}}, \tilde{t}), & p(\mathbf{x}, t) & =M^{\mathfrak{c}} \tilde{p}(\tilde{\mathbf{x}}, \tilde{t}), \\
\mathbf{g}(\mathbf{x}) & =\tilde{\mathbf{g}}(\tilde{\mathbf{x}}), &
\end{array}
$$

which leaves $\mathfrak{c}$ and $\mathfrak{b}$ arbitrary, but gives the relation $F r=M$. This choice will be taken in what follows. Note that it is dictated by the outside world and is not derivable from the system (4)-(6). Performing asymptotic expansions on all the quantities now yields

$$
\nabla p^{(0)}=\varrho^{(0)} \mathbf{g}^{(0)}
$$


which is the equation of hydrostatic equilibrium encountered in (28) already, and which is to be seen contrasting $(21)$.

\section{Finite volume methods For the LOW MACH NUMBER REGime}

\subsection{Finite volume methods for conservation laws}

For the general $n \times n$ hyperbolic system

$$
\partial_{t} q+\nabla \cdot f(q)=0 \quad q: \mathbb{R}_{0}^{+} \times \mathbb{R}^{d} \rightarrow \mathbb{R}^{n}, \quad f: \mathbb{R}^{n} \rightarrow \mathbb{R}^{n}
$$

of conservation laws in $d$ spatial dimensions a finite volume scheme is obtained by defining interface fluxes $\hat{f}_{i+\frac{1}{2}}$ which are used to update the cell values $q_{i}$ :

$$
\partial_{t} q_{i}+\frac{\hat{f}_{i+\frac{1}{2}}-\hat{f}_{i-\frac{1}{2}}}{\Delta x}=0
$$

Incompressible flows and thus the low Mach number limit simplify to trivial solutions in one-dimensional situations and therefore are of interest only in a multiple spatial dimensions (see [8]). For simplicity of notation the scheme (38) is given for a one-dimensional and equidistant grid, but the arguments presented in this section apply dimension by dimension to multi-dimensional schemes of the form

$$
\partial_{t} q_{i}+\frac{\hat{f}_{i+\frac{1}{2}, j}^{(x)}-\hat{f}_{i-\frac{1}{2}, j}^{(x)}}{\Delta x}+\frac{\hat{f}_{i, j+\frac{1}{2}}^{(y)}-\hat{f}_{i, j-\frac{1}{2}}^{(y)}}{\Delta y}=0
$$

and analogously in three dimensions.

A consistent numerical flux $\hat{f}_{i+\frac{1}{2}}$ should in particular ensure stability of the scheme when subject to explicit or implicit time integration. A possible way is, upon a local linearization to enforce upwinding for every characteristic variable. This yields the Roe scheme [21]

$$
\hat{f}_{i+\frac{1}{2}}=\frac{1}{2}\left[f\left(q_{i+1}\right)+f\left(q_{i}\right)\right]-\frac{1}{2}\langle|A|\rangle_{\mathrm{Roe}}\left(q_{i+1}-q_{i}\right)
$$

with $A=f^{\prime}$ the Jacobian of (37). Given its diagonalization $A=R \Lambda R^{-1},|A|$ is defined as $R|\Lambda| R^{-1}$. Finally, $|A|$ as function of $q$ is evaluated at an average state $\langle q\rangle_{\mathrm{Roe}}$. The second term in (40) is responsible for the stability of the scheme and adds the necessary diffusion. This enables the method to resolve shocks. In a numerical simulation of compressible flow at very low Mach numbers however this method is not able to maintain the features of an incompressible flow. [8,12] have given theoretical arguments to explain this experimental finding. In Section 3.3 we will repeat them. In short, excessive diffusion, scaling as $\mathcal{O}(1 / M)$ renders any attempt to resolve low Mach numbers with the Roe scheme unsuccessful.

\subsection{Modifications of diffusion matrices in Roe-type methods}

It has been found in [23] that replacing $|A|$ in (40) by a specially chosen different matrix $D$ leads to very much improved results regarding the ability of the scheme to cope with the incompressible limit. For historical reasons, these modifications have been called flux preconditioning. In this paper $D$ will be called a modified diffusion or upwinding matrix, consistently with [2]. The particular choice in [23] is $D=P^{-1}|P A|$ with $P$ 
from $[26]$

$$
P=\left(\begin{array}{ccccc}
1 & 0 & 0 & 0 & \frac{\mu^{2}-1}{c^{2}} \\
0 & 1 & 0 & 0 & 0 \\
0 & 0 & 1 & 0 & 0 \\
0 & 0 & 0 & 1 & 0 \\
0 & 0 & 0 & 0 & \mu^{2}
\end{array}\right) \quad D=\left(\begin{array}{ll} 
& \mathcal{O}\left(1 / M^{2}\right) \\
\mathcal{O}(1) & \mathcal{O}\left(1 / M^{2}\right) \\
\mathcal{O}\left(1 / M^{2}\right) \\
\mathcal{O}\left(1 / M^{2}\right)
\end{array}\right)
$$

given here in the basis of primitive variables. The parameter $\mu$ is given by $\mu=\min \left[1, \max \left(M_{\text {loc }}, M_{\text {cut }}\right)\right]$ and $M_{\text {cut }}$ avoids singularity of the matrix. Its value should be chosen smaller than the smallest expected relevant Mach numbers of the flow.

The performance of this scheme in the context of homogeneous equations (1)-(3) has been analyzed in [12]. The diffusion matrix is multiplied with the jumps $q_{i+1}-q_{i}$, and having a pressure background which is spatially constant up to terms of the order $\mathcal{O}\left(M^{2}\right)$ the result is $\mathcal{O}(1)$. This explains the lack of Mach number dependent diffusion as observed experimentally.

When applied to (4)-(6) however, the pressure (and equally the energy) is in lowest orders not spatially constant, but varies according to (36). Keeping the divergent terms in the density and energy rows would make them dominate the asymptotics, because the terms involving the physical flux and also the source terms do not contain explicit, and in particular negative, powers of $M$ in these rows. The limit equations will be given entirely by the diffusive part and will not reflect the properties of the physical limit (36). Thus the scheme will not be preserving the asymptotics in the combined limit $M \rightarrow 0, M / F r=1$.

Additionally to the scaling, applying this scheme to any stationary initial data which contain a non-constant pressure gradient, introduces terms which scale with the inverse of $M_{\text {cut }}$ (see e.g. [20]). Therefore the numerical errors strongly depend on an arbitrary parameter, and are unacceptable for low values of $M_{\text {cut }}$. In practice, the simulations crash after very short times.

To correct this behavior, in [19] a different matrix $P$ has been suggested. In entropy variables it takes the form

$$
P_{\text {entr }}=\left(\begin{array}{ccccc}
1 & n_{x} \delta & n_{y} \delta & n_{z} \delta & 0 \\
-n_{x} \delta & 1 & 0 & 0 & 0 \\
-n_{y} \delta & 0 & 1 & 0 & 0 \\
-n_{z} \delta & 0 & 0 & 1 & 0 \\
0 & 0 & 0 & 0 & 1
\end{array}\right)
$$

with $\delta=\frac{1}{\min \left(1, \max \left(M_{\mathrm{loc}}, M_{\mathrm{cut}}\right)\right)}-1$. In primitive variables it is

$$
P_{\text {prim }}=\left(\begin{array}{ccccc}
1 & n_{x} \frac{\varrho \delta M}{c} & n_{y} \frac{\varrho \delta M}{c} & n_{z} \frac{\varrho \delta M}{c} & 0 \\
0 & 1 & 0 & 0 & -n_{x} \frac{\delta}{\varrho c M} \\
0 & 0 & 1 & 0 & -n_{y} \frac{\delta}{\varrho c M} \\
0 & 0 & 0 & 1 & -n_{z} \frac{\delta}{\varrho c M} \\
0 & n_{x} \varrho c \delta M & n_{y} \varrho c \delta M & n_{z} \varrho c \delta M & 1
\end{array}\right) .
$$

In $x$-direction, the diffusion matrix then reads

$$
D=\frac{1}{M^{2}}\left(\begin{array}{ccccc}
0 & 0 & 0 & 0 & 0 \\
0 & 0 & 0 & 0 & \gamma-1 \\
0 & 0 & 0 & 0 & 0 \\
0 & 0 & 0 & 0 & 0 \\
0 & 0 & 0 & 0 & 0
\end{array}\right)+\mathcal{O}(1)
$$


Observe that now the $\mathcal{O}\left(1 / M^{2}\right)$ terms have disappeared from both the pressure and the density row such that a Roe-type scheme, when using (44), will not violate (36) in the limit of small Mach numbers when $M=F r$.

Contrary to $D$ as given in (41), additionally the new matrix in (44) has a finite limit for $M_{\text {cut }} \rightarrow 0$, although this parameter is still needed in the definition of $P$.

\subsection{Asymptotic analysis}

It is important for the scheme to give good results for both the homogeneous equations and those with gravity source terms. Often gravity only acts in one direction, such that there the limit equation (36)

$$
\nabla p^{(0)}=\varrho^{(0)} \mathbf{g}^{(0)}
$$

has to be obtained, whereas in all the other directions (21)

$$
\nabla p^{(0)}=0=\nabla p^{(1)}
$$

is the correct one. To highest order the new scheme gives

$0=\partial_{t}\left(\begin{array}{c}\varrho \\ v^{x} \\ v^{y} \\ v^{z} \\ e\end{array}\right)_{i}+\frac{1}{2 \Delta x}\left[\frac{1}{M^{2}}\left(\begin{array}{c}0 \\ p_{i+1}-p_{i-1} \\ 0 \\ 0 \\ 0\end{array}\right)-\frac{\gamma-1}{M^{2}}\left(\begin{array}{c}0 \\ e_{i+1}-2 e_{i}+e_{i-1} \\ 0 \\ 0 \\ 0\end{array}\right)\right]+$ other directions $+\mathcal{O}(M)+$ source

The two lowest orders can be simplified (for $\ell=0,1$ one has $\left.p^{(\ell)}=(\gamma-1) e^{(\ell)}\right)$ to formally yield in the limit $M \rightarrow 0$ :

$$
\frac{p_{i}^{(\ell)}-p_{i-1}^{(\ell)}}{\Delta x}=\text { source } \quad \ell=0,1 .
$$

The rest of the asymptotic analysis is done with the $\mathcal{O}(1)$ equations, which due to the consistency of the scheme gives consistent discretizations of the remaining equations in the limit $M \rightarrow 0$. In view of the findings we expect our scheme for the homogeneous system to have pressure perturbations $\mathcal{O}\left(M^{2}\right)$, consistently with the results at the continuous level (Sect. 1.2). Therefore this scheme still is able to solve the homogeneous Euler equations, but contrary to (41) has the right asymptotics in the combined low Mach/low Froude number limit, which has been identified as the one most important for simulations of stratified atmospheres. In particular, the absence of divergent terms in those rows where the physical flux, or the gravity source terms, do not have explicit $M$ dependence prevents the diffusion, which to a certain extent is arbitrary, to dominate the asymptotics.

It is interesting to compare this analysis to the Roe scheme when no modifications are applied. As has been mentioned before, the Roe scheme does not yield satisfactory results when applied to a low Mach number situation. For the homogeneous system, equation (45) for the Roe solver is

$$
\begin{aligned}
& \frac{p_{i+1}^{(0)}-p_{i-1}^{(0)}}{\Delta x}=0 \\
& \frac{p_{i+1}^{(1)}-p_{i-1}^{(1)}}{\Delta x}=\Delta x \cdot\left(\text { terms involving } 2^{\text {nd }} \text { derivatives of } \varrho, v, e\right)+\mathcal{O}\left(\Delta x^{2}\right) .
\end{aligned}
$$

Although it is also a discretization of $\nabla p^{(\ell)}=0$, it is not a good approximation for finite values of $\Delta x$ - contrary to (45). It is always possible to cure the low Mach number problems by increasing the resolution. This however is both impractical and unnecessary. 
Note that the correct asymptotic scaling in general does not guarantee that for finite resolution the numerical solution will be close to the analytical one. The situation is somewhat easier in the homogeneous case, where any solution to the equation $\nabla p^{(0)}=0$ can be represented exactly on a numerical grid. A discrete version of an exact solution to $\nabla p^{(0)}=\varrho^{(0)} \mathbf{g}^{(0)}$ with $\mathbf{v}=0$ will in general not remain stationary in a numerical simulation because of the mismatch between the exact derivative and the way its numerical approximation is obtained from adjacent cell averages. The correct asymptotics however means that the errors do not increase without bound in the limit of small Mach numbers. If, in addition to the correct scaling in the vicinity of a hydrostatic equilibrium, one wishes to be able to maintain the equilibrium itself exactly stationary (up to machine precision), a specific discretization (well-balancing) of the source term will be necessary (e.g. [4]). To combine the two approaches is subject of ongoing work.

\section{Time integration}

\subsection{Stability with explicit time integration}

The improved performance of the scheme in the low Mach number limit was achieved by modifying the artificial upwind viscosity matrix - a term that was introduced to stabilize the scheme. This raises the question of the stability of the resulting new method when subject to explicit time discretization.

The investigation of linear stability with the von Neumann method yields results on the time behavior of Fourier modes for a linearized scheme. If all of the modes are damped in time, the method is called linearly stable. Surely, it is of relevance that the method is stable already in one spatial dimension and when integrated in time by a first order method. For simplicity, the following stability analysis is performed with piecewise constant reconstruction, i.e. on a method that is both spatially and temporally first order.

Every quantity $q_{i}^{n}$ is rewritten as a Fourier series in space $\left({ }^{\circ}=\sqrt{-1}\right)$ :

$$
q_{i}^{n}=\sum_{k \in \mathbb{Z}} q^{n} \exp \left({ }^{\circ} i k \Delta x\right) .
$$

Insert this into the fully discrete scheme $\left(\nu=\frac{\Delta t}{\Delta x}\right)$

$$
q_{i}^{n+1}=q_{i}^{n}-\frac{1}{2} \nu\left[A\left(q_{i+1}^{n}-q_{i-1}^{n}\right)-D\left(q_{i+1}^{n}-2 q_{i}^{n}+q_{i-1}^{n}\right)\right]
$$

to obtain, by defining $k \Delta x=: \beta$,

$$
q^{n+1}=\left\{\mathbb{1}-\nu\left[A_{0} \sin \beta+D(1-\cos \beta)\right]\right\} q^{n}
$$

The expression in curly brackets is called amplification matrix. Stability of such iterated linear maps needs all its eigenvalues to be less than 1 in absolute value.

A detailed stability analysis is performed in [2]. Evaluating the limit $M \rightarrow 0$ for the suggested upwinding matrix yields

$$
\nu_{\max } \sim \frac{M}{c} \frac{\sqrt{1+\delta^{2}}}{\delta^{2}} \in \mathcal{O}\left(M^{2}\right)
$$

Contrary to the result $\nu_{\max } \in \mathcal{O}(M)$ valid e.g. for the Roe scheme, (51) implies stability under a more severe CFL condition. This result is known to hold for the scheme proposed in [23] as well (see [3]). For the scheme proposed here, a stable explicit integration in time is therefore possible, but makes the implementation of an implicit method as described in [19] favorable. 


\section{Numerical RESUlts}

\subsection{Incompressible vortex}

In order to test the ability of the new scheme to resolve flows of low Mach number when applied to the homogeneous Euler equations, in Fig. 1 we show the evolution of an incompressible vortex from [11].
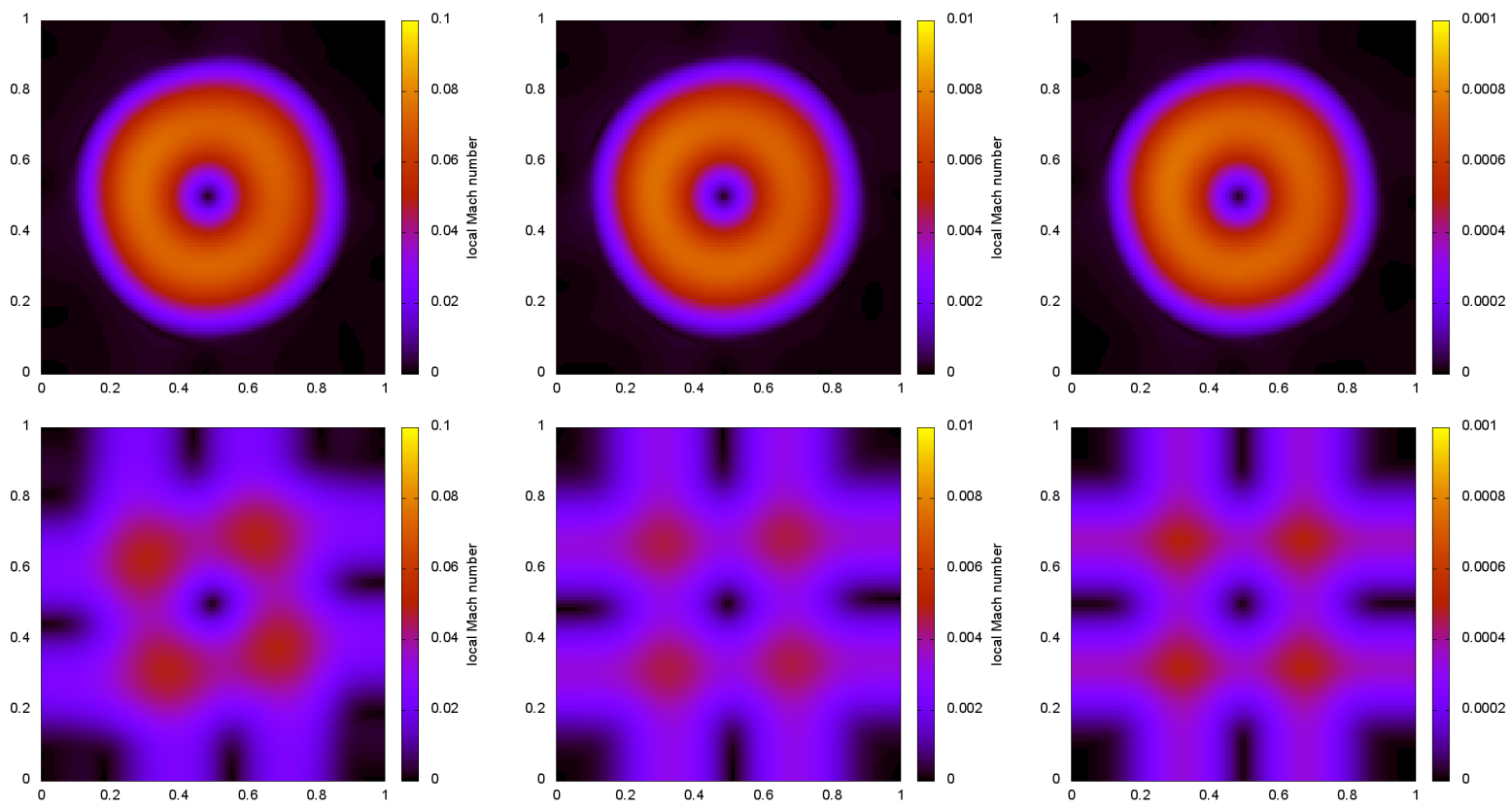

Figure 1. Results of simulations of the Gresho vortex up to a time of 0.8 , colour coded is the local Mach number. The upper row shows the results obtained with the solver from [19], the lower row has been calculated with the Roe solver; from left to right $M$ is $0.1,0.01$ and 0.001 , respectively.

Its density is uniformly constant and the rotation speed is given by

$$
v(r)= \begin{cases}5 r & r<0.2 \\ 2-5 r & 0.2 \leq r<0.4 \\ 0 & r \geq 0.4\end{cases}
$$

from which the pressure follows up to an arbitrary additive constant. This constant is chosen $\mathcal{O}\left(1 / M^{2}\right)$ and the Figure displays the local Mach number of the vortex at $t=0.8$ for $M \in\{0.1,0.01,0.001\}$ for both the new solver introduced in [19] and discussed here and the unmodified Roe solver. The setup is solved on a grid of $100 \times 100$ cells with a Runge-Kutta method of $3^{\text {rd }}$ order with a piecewise constant reconstruction.

Comparison of the images shows a numerical diffusion which is virtually independent of the Mach number when the modified diffusion matrix (44) is used, whereas the diffusion of the Roe solver, even for the moderate Mach number of 0.1 , has destroyed the vortex entirely. This confirms the correct scaling of the method in the limit $M \rightarrow 0$ of vanishing Mach number for the homogeneous Euler equations.

\subsection{Isothermal atmosphere}

As a further test we choose an isothermal hydrostatic equilibrium of an ideal gas with $\gamma=1.4$, given by (28) and $p / \varrho=1$. The test is performed in one spatial dimension with gravity pointing towards negative values of 
the spatial coordinate with a computational domain of $[0,1]$. It is discretized with $N \in\{50,100,200,400\}$ cells. We use a Runge-Kutta scheme of $3^{\text {rd }}$ order, a piecewise constant reconstruction and fix the values in the ghost cells to their initial values.

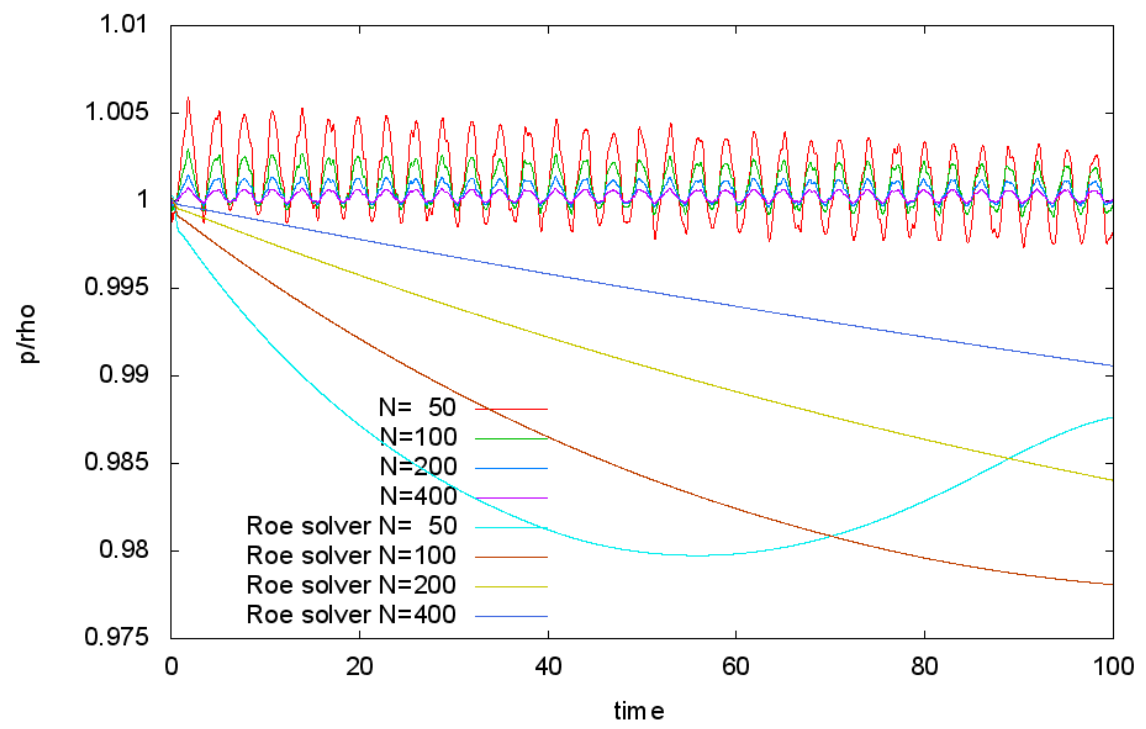

FIGURE 2. Numerical evolution for the initial data of an isothermal, stationary atmosphere. $p / \varrho$, which is proportional to the temperature, is shown as a function of time at a fixed location of $x=0.85$. For comparison, additionally to the results computed with the new scheme, the evolution of the same setup with the Roe solver is shown.

The exact solution for this setup is stationary. The numerical results are displayed in Fig. 2, where $p / \varrho$, which is proportional to temperature, is shown as a function of time at a fixed position of $x=0.85$. An oscillation is observed, which does not change significantly with time, but whose amplitude decreases with spatial resolution. Its origin is the fact that the numerical discretization of the pressure gradient is not exactly balanced by the numerical treatment of the source term, which is evaluated in a cell-centered manner. The residual acts as a perpetual excitation of the atmosphere to which it answers by oscillation. The characteristic frequency is of the order of the Brunt-Väisälä frequency of this setup. As expected, the numerical errors in the approximation of the gradient and therefore the perturbations decrease with the resolution.

For comparison, the Figure shows the time evolution of the same initial data of the isothermal equilibrium with the unmodified Roe solver. It suffers from a similar kind of mismatch between the numerical approximation to the gradient and the source term, but the perturbations experience the strong diffusion which damps oscillations and manifests itself in a rapid decrease of the temperature. In principle, again the deviations can be controlled by increasing the resolution although the diffusive character persists.

\section{Conclusions}

The ability to resolve the low Mach number limit $M \rightarrow 0$ of ideal hydrodynamics is considered an important step towards improving the accuracy of numerical methods. For the homogeneous Euler equations the limit consists of a spatially constant pressure up to perturbations $\mathcal{O}\left(M^{2}\right)$. When gravity source terms are taken into account, the gradient of the lowest order term of an expansion of the pressure in powers of $M$ has to balance the lowest order of the gravity. This additional feature has been found to contradict the scheme proposed in $[23,26]$, which is a modified Roe-type scheme able to resolve the low Mach number limit for the homogeneous 
system. Here we have analyzed the shortcomings and justified the ability of the modification proposed in [19] to comply with both the low Mach number limit in the homogeneous case and in presence of gravity by means of an asymptotic analysis. In the latter case the combined limit $M \rightarrow 0, M=F r$ has been found to be the most relevant one. We have demonstrated the absence of an excessive diffusion for this method in the limit of small Mach numbers and shown experimentally that it preserves a hydrostatic equilibrium up to perturbations due to the treatment of the gravity source term. However these perturbations again do not show the diffusion observed with the Roe solver.

Further work aims at combining this method with a well-balancing procedure which reduces the mismatch between the numerical approximation to the gradient of the pressure and the numerical treatment of the gravity source term. The combined method will allow to resolve hydrostatic equilibria and the low Mach number motion of a stratified gas to very high accuracy.

\section{REFERENCES}

[1] Kiyoshi Asano, On the incompressible limit of the compressible euler equation, Japan Journal of Applied Mathematics 4 (1987), no. 3, 455-488.

[2] Wasilij Barsukow, Philipp V. F. Edelmann, Christian Klingenberg, Fabian Miczek, and Friedrich K. Röpke, A numerical scheme for the compressible low-mach number regime of ideal fluid dynamics, University of Wuerzburg (preprint, 2016), available as http://tinyurl.com/zluw3gt.

[3] Philipp Birken and Andreas Meister, Stability of preconditioned finite volume schemes at low mach numbers, BIT Numerical Mathematics 45 (2005), no. 3, 463-480.

[4] Praveen Chandrashekar and Christian Klingenberg, A second order well-balanced finite volume scheme for euler equations with gravity, SIAM Journal on Scientific Computing 37 (2015), no. 3, B382-B402.

[5] Phillip Colella and Paul R Woodward, The piecewise parabolic method (ppm) for gas-dynamical simulations, Journal of computational physics 54 (1984), no. 1, 174-201.

[6] Floraine Cordier, Pierre Degond, and Anela Kumbaro, An asymptotic-preserving all-speed scheme for the euler and navierstokes equations, Journal of Computational Physics 231 (2012), no. 17, 5685-5704.

[7] Richard Courant, Kurt Friedrichs, and Hans Lewy, Über die partiellen differenzengleichungen der mathematischen physik, Mathematische Annalen 100 (1928), no. 1, 32-74.

[8] Stéphane Dellacherie, Analysis of godunov type schemes applied to the compressible euler system at low mach number, Journal of Computational Physics 229 (2010), no. 4, 978-1016.

[9] David G Ebin, The motion of slightly compressible fluids viewed as a motion with strong constraining force, Annals of mathematics (1977), 141-200.

[10] Sergei Konstantinovich Godunov, A difference method for numerical calculation of discontinuous solutions of the equations of hydrodynamics, Matematicheskii Sbornik 89 (1959), no. 3, 271-306.

[11] Philip M Gresho and Stevens T Chan, On the theory of semi-implicit projection methods for viscous incompressible flow and its implementation via a finite element method that also introduces a nearly consistent mass matrix. part 2: Implementation, International Journal for Numerical Methods in Fluids 11 (1990), no. 5, 621-659.

[12] Hervé Guillard and Cécile Viozat, On the behaviour of upwind schemes in the low mach number limit, Computers \& fluids $\mathbf{2 8}$ (1999), no. 1, 63-86.

[13] Amiram Harten, Peter D Lax, and Bram van Leer, On upstream differencing and godunov-type schemes for hyperbolic conservation laws, SIAM review 25 (1983), no. 1, 35-61.

[14] Hiroshi Isozaki, Wave operators and the incompressible limit of the compressible euler equation, Communications in mathematical physics 110 (1987), no. 3, 519-524.

[15] Sergiu Klainerman and Andrew Majda, Singular limits of quasilinear hyperbolic systems with large parameters and the incompressible limit of compressible fluids, Communications on Pure and Applied Mathematics 34 (1981), no. 4, 481-524.

[16] R Klein, Semi-implicit extension of a godunov-type scheme based on low mach number asymptotics i: one-dimensional flow, Journal of Computational Physics 121 (1995), no. 2, 213-237.

[17] H-O Kreiss, J Lorenz, and MJ Naughton, Convergence of the solutions of the compressible to the solutions of the incompressible navier-stokes equations, Advances in Applied Mathematics 12 (1991), no. 2, 187-214.

[18] Guy Métivier and Steve Schochet, The incompressible limit of the non-isentropic euler equations, Archive for rational mechanics and analysis 158 (2001), no. 1, 61-90.

[19] F Miczek, FK Röpke, and PVF Edelmann, New numerical solver for flows at various mach numbers, Astronomy \& Astrophysics 576 (2015), A50.

[20] Fabian Miczek, Simulation of low mach number astrophysical flows, Ph.D. thesis, Technische Universität München, Dissertation, 2013. 
[21] Philip L Roe, Approximate riemann solvers, parameter vectors, and difference schemes, Journal of computational physics $\mathbf{4 3}$ (1981), no. 2, 357-372.

[22] Steven Schochet, Fast singular limits of hyperbolic pdes, Journal of differential equations 114 (1994), no. 2, 476-512.

[23] Eli Turkel, Preconditioned methods for solving the incompressible and low speed compressible equations, Journal of computational physics 72 (1987), no. 2, 277-298.

[24] Seiji Ukai et al., The incompressible limit and the initial layer of the compressible euler equation, Journal of Mathematics of Kyoto University 26 (1986), no. 2, 323-331.

[25] Bram Van Leer, Towards the ultimate conservative difference scheme. v. a second-order sequel to godunov's method, Journal of computational Physics 32 (1979), no. 1, 101-136.

[26] Jonathan M Weiss and Wayne A Smith, Preconditioning applied to variable and constant density flows, AIAA journal 33 (1995), no. 11, 2050-2057. 